\title{
Skill and Skill Prediction of Cloud-Track Advection-Only Forecasting under a Cumulus-Dominated Regime $\mathscr{O}$
}

\author{
VIVEK SRIKRISHNAN \\ Department of Energy and Mineral Engineering, The Pennsylvania State University, \\ University Park, Pennsylvania \\ GEORGE S. YOUNG \\ Department of Meteorology and Atmospheric Science and Department of Energy and Mineral Engineering, \\ The Pennsylvania State University, University Park, Pennsylvania \\ JEFFREY R. S. BROWNSON \\ Department of Energy and Mineral Engineering, The Pennsylvania State University, \\ University Park, Pennsylvania
}

(Manuscript received 20 June 2016, in final form 30 September 2016)

\begin{abstract}
The intermittency of solar power production is dependent on the evolution and advection of the nearby cloud field. A key problem related to solar energy integration is the improvement of 1-h-ahead forecasts to reduce the impact of intermittency on power systems operations. Many solar forecasts explicitly or implicitly assume Taylor's hypothesis. While such advection-only forecasts can be presumed to be valid across sufficiently short time scales, it is not clear how rapidly the skill of such a forecast decays with increased lead time. As the goal is to improve the quality of 1-h-ahead forecasts, this work focuses on quantifying the skill of cloudtrack wind-based cumulus-dominated cloud field forecasts with respect to lead time. No explicit connection is drawn to the quality of solar forecasts because of the importance of separating two potential sources of error: cloud field forecasting and radiative transfer estimation. It is found that the cumulus field forecast skill begins to asymptotically approach a minimum at lead times of beyond $30 \mathrm{~min}$, suggesting that advection-only forecasts in a cumulus-dominated environment should not be relied upon for 1-h-ahead point forecasts used by radiative transfer methods to estimate solar power production. A first attempt at forming a probabilistic forecast that can quantify this increasing uncertainty when using advection-only methods is presented.
\end{abstract}

\section{Introduction}

Renewable sources of energy (e.g., wind and solar) are intermittent, which can result in significant operational challenges given a large market share (penetration) of energy generation (Von Meier 2011; Eber and Corbus 2013). Clouds are the major source of difficult-to-predict solar energy intermittency, affecting both the overall availability of the solar resource and the ratio between the

Supplemental information related to this paper is available at the Journals Online website: http://dx.doi.org/10.1175/JAMC-D16-0224.s1.

Corresponding author e-mail: Vivek Srikrishnan, vxs914@psu. edu direct normal irradiance and diffuse components of shortwave light. As a result, accurate forecasting of the cloud field over a variety of time scales (from seconds to days) is crucial to properly manage an energy portfolio including a group of assets that function as solar energy conversion systems (Von Meier 2011; Ela et al. 2013).

Various strategies have been studied to address the operational difficulties associated with high levels of solar technology penetration (e.g., photovoltaics and concentrating solar power) (Ela et al. 2013; Eber and Corbus 2013). Ela et al. (2013) suggested that accurate 1-h-ahead forecasts would mitigate the effect of solar resource variability on power imbalance (a significant difference between generated and demanded power). Many current approaches to solar forecasting with lead times less than $3-5 \mathrm{~h}$ assume a frozen cloud field, which develops only 
through advection. The lead times for these forecasts may be on the order of seconds and minutes using a total sky imager (Chow et al. 2011; Huang et al. 2013; Marquez and Coimbra 2013; Quesada-Ruiz et al. 2014), other groundbased imaging equipment (Yang et al. 2014; Chow et al. 2015), or satellite imagery (Miller et al. 2012). In Perez et al. (2010), 1-6-h-ahead forecasts are obtained using an advection-only technique based on satellite images transformed into a measure of radiance attenuation. A review of the range of methodologies and results from advectiononly direct normal irradiance forecast studies can be found in Law et al. (2014, section 6).

However, particularly for longer lead times, it is often unclear how much of the forecast error is due to a misforecast of the cloud field and how much is due to uncertainty in the radiative transfer model applied to that field to derive irradiance forecasts [an analysis of the error of satellite imagery-to-irradiance methods is carried out in Law et al. (2014, section 6.2)]. Decoupling these two sources of error (cloud field misforecast vs radiative transfer model) is important for diagnosing the causes of error and therefore improving the quality of irradiance and solar power forecasts. The current study will focus solely on errors in cloud field forecasting.

The frozen field hypothesis (also known as Taylor's hypothesis) is commonly invoked and studied in applications of fluid dynamics (Gifford 1956; Powell and Elderkin 1974; Mizuno and Panofsky 1975; Wacongne and Babiano 1982; Pollard et al. 2000; Higgins et al. 2012). In solar power forecasting, Taylor's hypothesis is used to justify the use of advection-only cloud models. As originally formulated by Taylor (1938, p. 478), the assumption reads: "If the velocity of the air stream which carries the eddies is very much greater than the turbulent velocity," then local changes are the result of advection alone. This is equivalent to assuming that the feature of interest (e.g., a cloud) blows past a site faster than it evolves. This hypothesis may be represented formally as

$$
q(\Delta t, \mathbf{p}+\Delta t \mathbf{v})=q(0, \mathbf{p})
$$

where $q$ is the quantity of interest, represented as a function of time $t$ and spatial position $\mathbf{p}$, and $\mathbf{v}$ is the mean advection velocity over the time interval $(0, \Delta t)$. (Definitions of all variables used in this paper are given in the appendix.) Taylor's hypothesis provides an important tool for linking the Lagrangian and Eulerian frames of reference for a flow, and as such, its validity has been studied extensively in fluid dynamics (e.g., Burghelea et al. 2005; Dennis and Nickels 2008; Del Álamo and Jiménez 2009; Wilczek et al. 2014) and humidity and rainfall (e.g., Zawadzki 1973; Gupta and Waymire 1987; Cox and Isham 1988; Li et al. 2009; Higgins et al. 2012).
In general, Eq. (1) will not be valid over all time scales, though it may provide a good approximation for sufficiently short $\Delta t$. Zawadzki (1973) concluded that Taylor's hypothesis for rainfall totals should not be applied beyond $40 \mathrm{~min}$. Higgins et al. (2012) determined through a field test and a large-eddy simulation that small-scale humidity features could be approximated by models assuming Taylor's hypothesis for sufficiently high spacetime correlations.

The scales that are relevant for the current study are illustrated in Fig. 1. In particular, the temporal scale (using a Lagrangian frame of reference) associated with cumulus cloud lifetimes is relevant for improving the quality of 1-h-ahead solar power forecasts, as recommended by Ela et al. (2013). As such, we are interested in understanding the extent to which Taylor's hypothesis holds for forecasts of cumulus-dominated cloud regimes. We will focus our attention on cloud forecasts, as Taylor's hypothesis is specifically related to that component of an overall solar forecast, and errors in forecasting the cloud field will propagate downstream to produce additional errors in the radiative transfer model.

\section{Methodology}

The overall methodology for the advection-only forecasting system used in this study is laid out in Fig. 2. Two visible channel satellite images, corresponding to the forecast time $t$ and a previous image from time $t-15 \mathrm{~min}$, are used to identify the advection (or cloud-track wind) vector field, which is defined over the study area and divided into blocks, as described in section $2 \mathrm{a}$. The wind vector field estimation procedure is detailed in section $2 b$. In light of the typical lifetime of fair-weather cumuli (as quantified in Fig. 1), the maximum lead time (or forecast horizon) for the forecasting step was restricted to $75 \mathrm{~min}$. A larger-scale study would be required to quantify the skill decay of advection-based forecasts for more slowly evolving synoptic-scale features.

\section{a. Data}

The data used in this study were obtained from the imager sensor of the NOAA GOES-13 geosynchronous satellite, also known as GOES-East, which has six bands, allowing for detection of visible radiation, longwave infrared radiation, shortwave infrared radiation, and water vapor. Data from the visible channel of the GOES imager, with a resolution of $1 \mathrm{~km}$ per pixel (the highest resolution available), were used in this study. Images from both the continental United States (CONUS) and extended Northern Hemisphere scans were used, allowing for 15-min intervals between images 


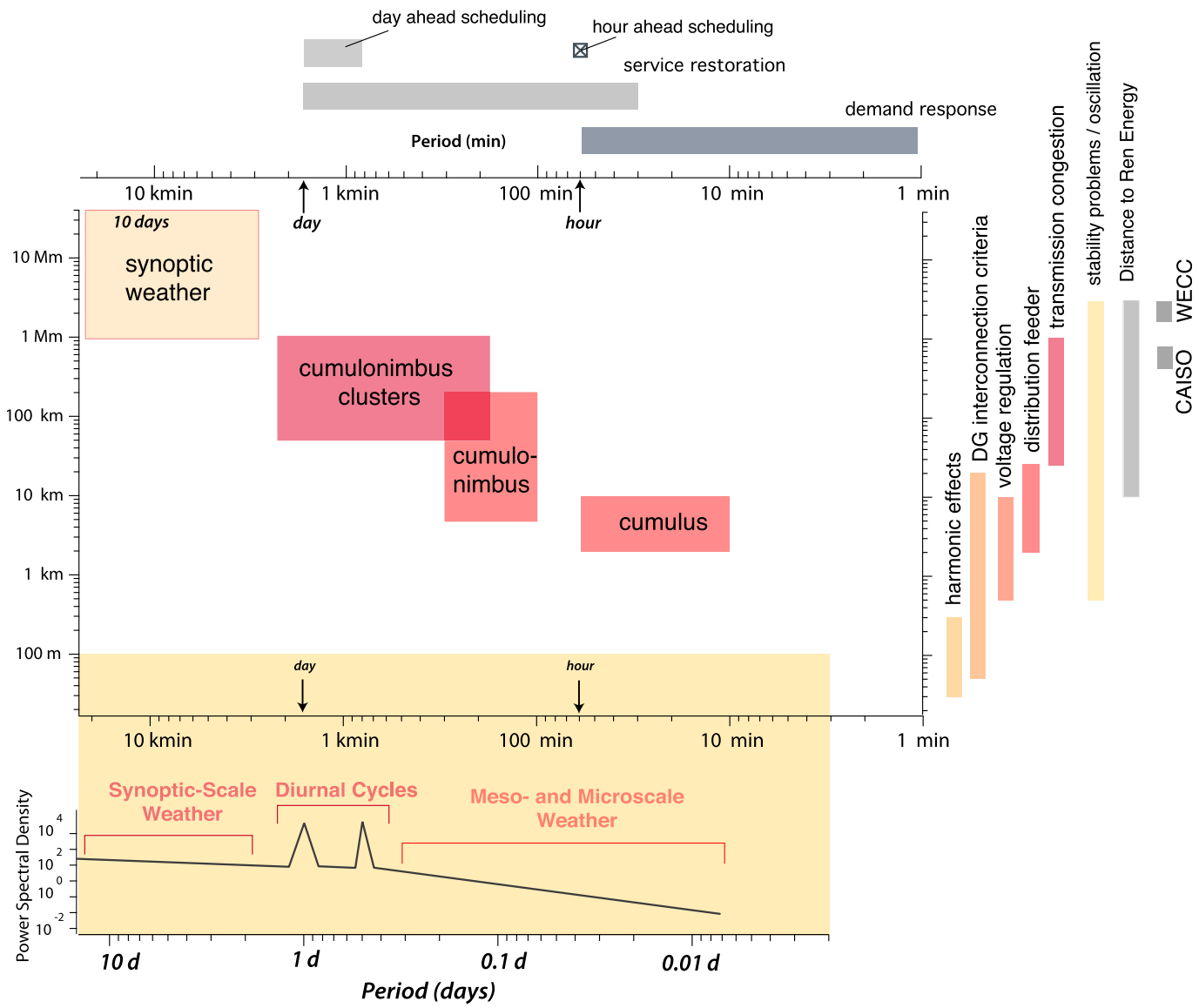

FIG. 1. Spatial and temporal scales of weather adapted from Rayl et al. (2013). The features and scales are based on data from Fujita (1981) and are illustrated along with power systems data from Von Meier (2011) and power spectral density.

[the choice of 15-min averaging times for low-level cloud-track winds follows Dunion and Velden (2002)].

The study area covers latitudes $34.83^{\circ}-42.65^{\circ} \mathrm{N}$ and longitudes $73.27^{\circ}-81.28^{\circ} \mathrm{W}$, essentially covering the midAtlantic region from North Carolina to Pennsylvania. The dataset covered the date range from 1 June to 31 August 2014 and was limited to the daylight hours of 1200-2100 UTC so that visible imagery could be used. The study area and period is representative of moist continental midlatitude summer, typically featuring a synoptically modulated cumulus-dominated cloud regime. This region also exhibits substantial variation in the underlying terrain, with mountains and seas as well as plateaus and plains. As a result, this forecasting challenge provides something of a "worst case" for advection-based forecasting.

Data from the visible channel of the GOES imager are in the form of 10-bit "raw" GOES Variable format (GVAR) counts, which have a linear relationship to the visible radiance of the area represented by a given pixel (which is in turn proportional to the visible albedo).
Many applications of cloud-track forecasting to solar irradiance have used a "cloud index" derived from these counts (e.g., Cano et al. 1986; Hammer et al. 1999; Perez et al. 2002; Marquez and Coimbra 2013; Mathiesen et al. 2013; Quesada-Ruiz et al. 2014; Cros et al. 2014; Law et al. 2014). However, the raw GVAR counts were used for this study as they are the fundamental measured quantities from which the cloud index and other quantities, such as radiance or reflectivity, are derived. The satellite measurements were corrected to account for first-order projection effects due to variations in the solar zenith angle (Perez et al. 2002). As these raw sensor measurements were used, each pixel was divided by the cosine of the zenith angle as calculated based on the time of the image and latitude and longitude of the pixel. This normalization eased the advection vector estimation procedure described in section $2 b$ by making the mean pixel values more comparable rather than drifting based on the diurnal solar cycle.

After the cosine correction of the solar zenith angle, images were subjected to a cloud masking method to 

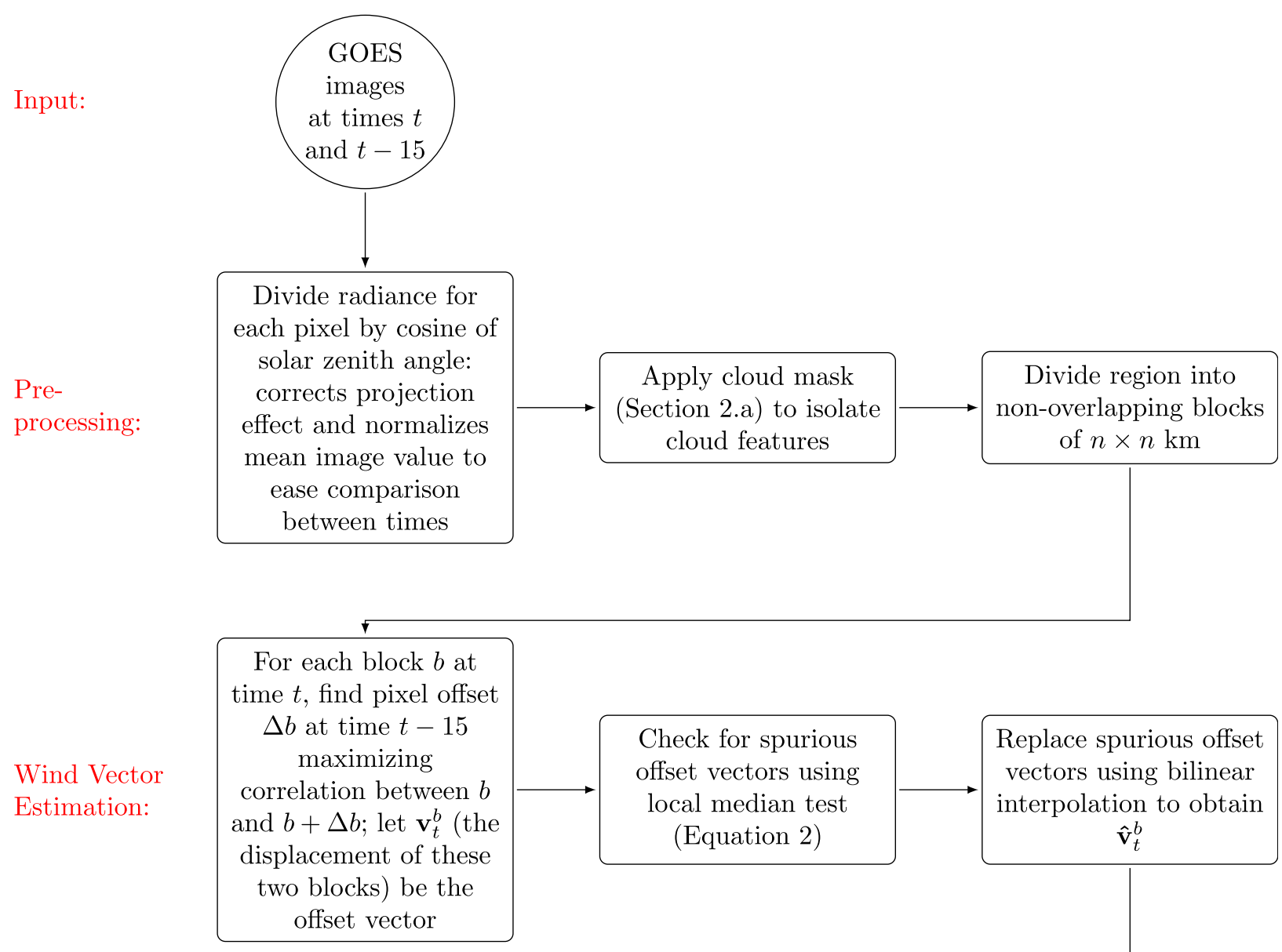

Wind Vector

Estimation:

Forecast:

Output:

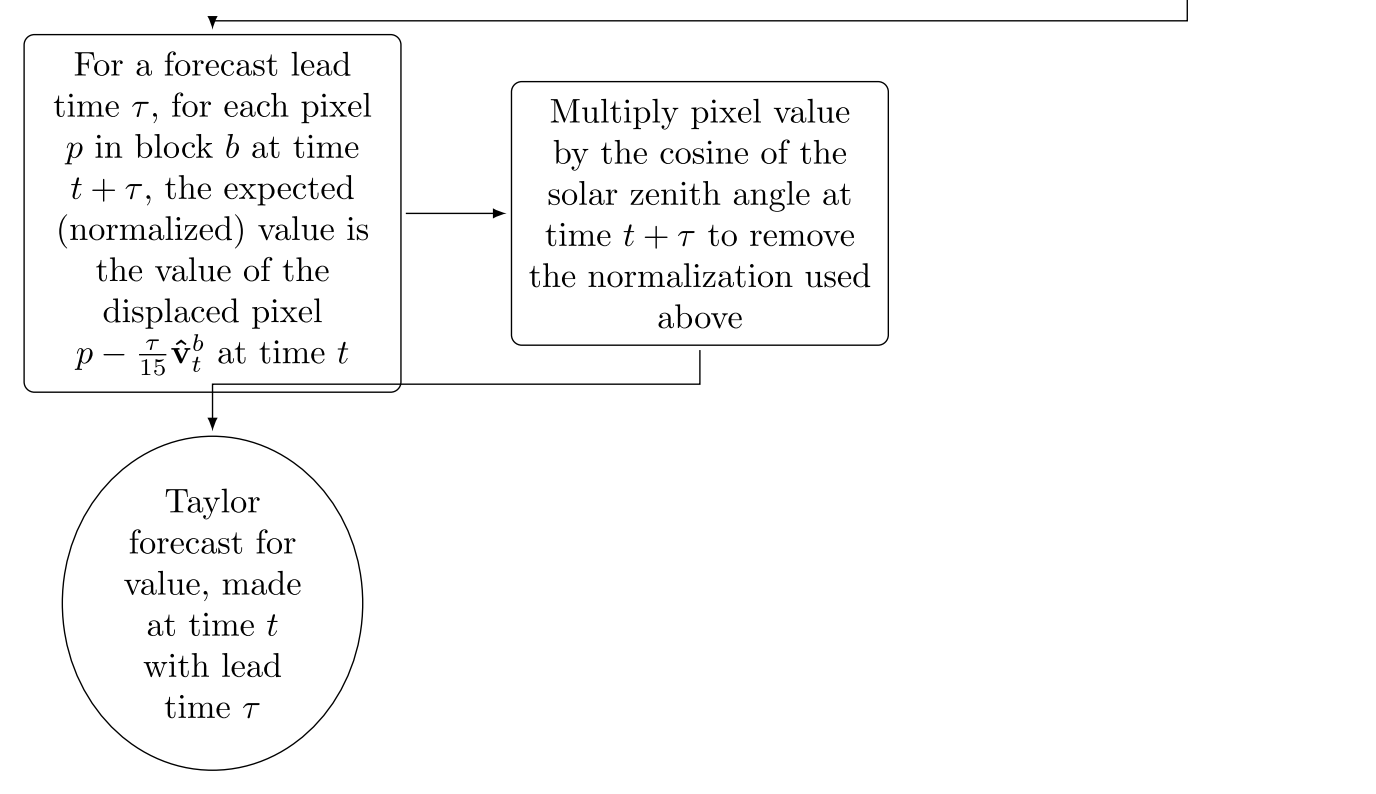

FIG. 2. Flowchart of the advection-only radiance forecasting method used in this study. Blocks $(b, a)$ and pixels $(x)$ are not indexed by time as they are treated as fixed geographic locations (or areas) rather than being time-dependent entities. 

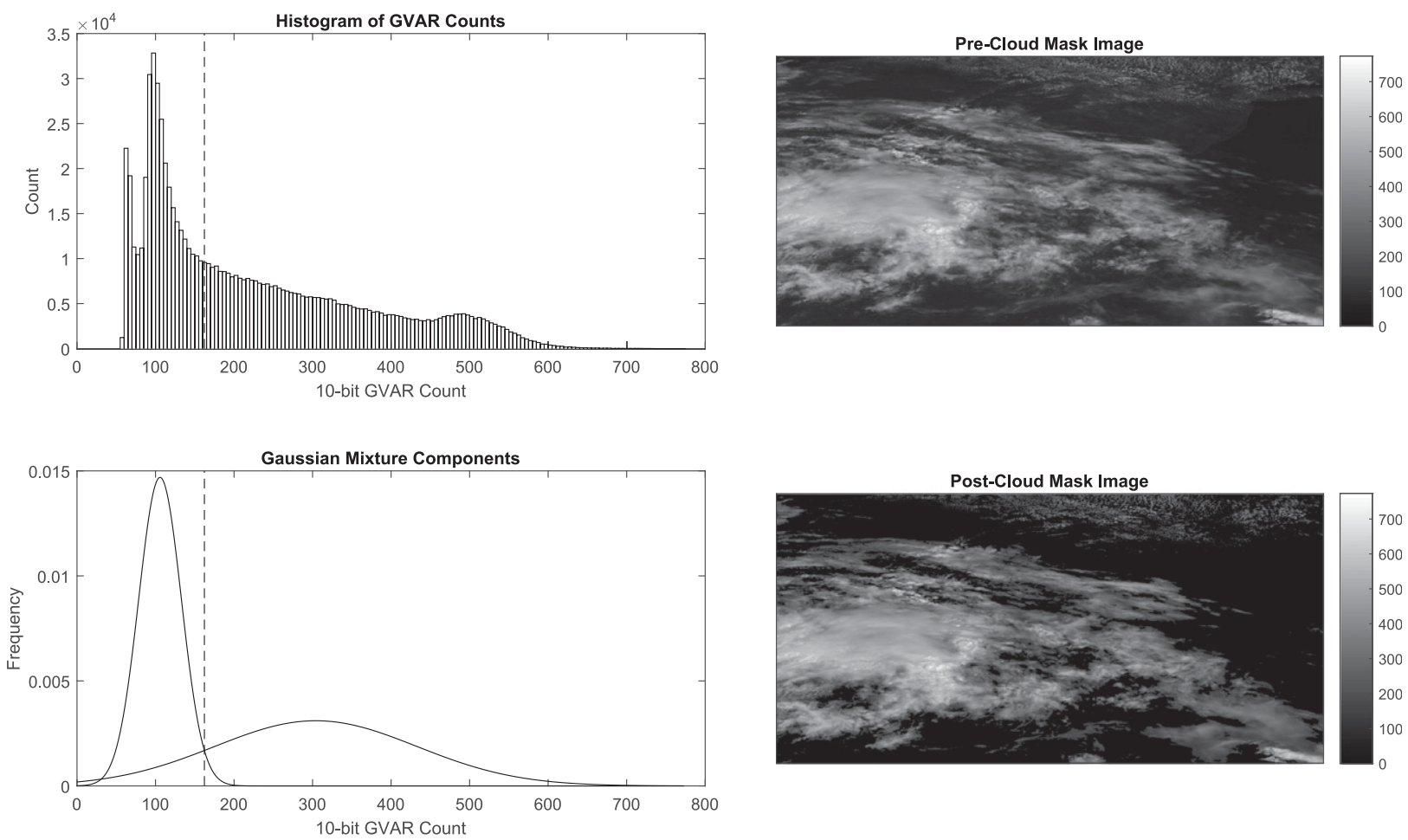

FIG. 3. Example application of the cloud mask procedure from section 2a. (left) The original data histogram and components of the fitted Gaussian mixture are presented along with the clear vs cloudy threshold (dashed line), beneath which the pixel values were zeroed. (right) The pre-cloud mask image and post-cloud mask image are also presented to show the resulting differences.

isolate cloud features. Based on the assumption that the count distribution of each image was generated by two underlying Gaussian distributions (one corresponding to "clear" pixels and one corresponding to "cloudy" pixels), a two-component Gaussian mixture model (McLachlan and Peel 2000) was fit to each image so that the fitted distribution of counts for each image had the form

$$
p N\left(\mu_{\text {clear }}, \sigma_{\text {clear }}\right)+(1-p) N\left(\mu_{\text {cloudy }}, \sigma_{\text {cloudy }}\right) \text {, }
$$

where $0 \leq p \leq 1$ is the mixing ratio of the two Gaussian distributions. The parameters in Eq. (2) are typically inferred by maximizing the likelihood function, either directly or using an expectation-maximization (EM) algorithm (McLachlan and Peel 2000), but may also be estimated using Bayesian methods (Früwirth-Schnatter 2006). The assumption that the overall pixel distribution is generated by two Gaussians is plausible given the study area and time period because of the lack of pixels corresponding to snow-covered areas. Pixels were deemed to belong to the clear mode of the mixture if their count values were no greater than the value at which the tails of the two Gaussians crossed. These pixels then had their value set to 0 to further sharpen the cloud feature boundaries. An example demonstrating the use of this cloud masking procedure, including the Gaussian mixture fit, can be seen in Fig. 3.

\section{b. Advection vector estimation and forecast generation}

Advection vectors were estimated over a grid of cells obtained by dividing each image into nonoverlapping $75 \mathrm{~km} \times 75 \mathrm{~km}$ regions. The cell size was chosen to get an adequate sample of the cumulus field for computing the correlation while still allowing for mesoscale and synoptic-scale variation in the wind field. As seen in Fig. 1, cells of this size allow for a sampling of multiple cumulus features as well as small-to-medium cumulonimbus features.

If Taylor's hypothesis [Eq. (1)] held perfectly, the correlation between the original and advected cells would be $r \approx 1$. However, this is unlikely to occur in practice given cumulus evolution, pixelation, and bidirectional radiance effects, so clouds were tracked by maximizing the correlation between pixel-displaced cells (at $t=\tau-15$ ) and the original (at $t=\tau$ ) cell within a given displacement search radius. Pixel offsets within a radius corresponding to a maximum mean advection speed of $55.6 \mathrm{~m} \mathrm{~s}^{-1}\left[50 \mathrm{~km}(15 \mathrm{~min})^{-1}\right]$, which is 

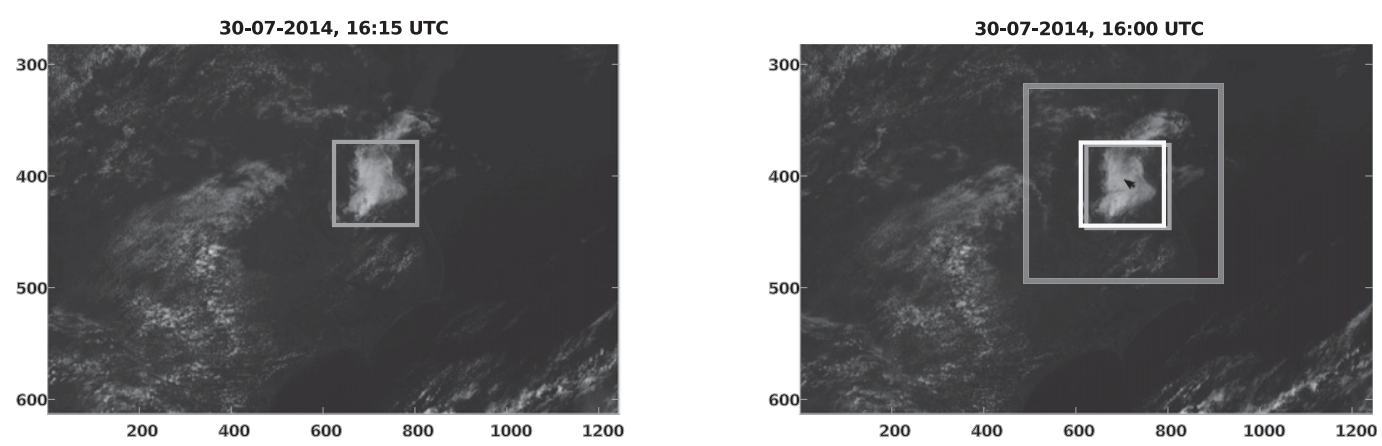

FIG. 4. Example of advection vector estimation: a forecast-time GOES image portion for (left) 1615 and (right) 1600 UTC. To obtain an estimate of the advection vectors used in forecasting, pixel offsets within a search radius corresponding to a maximum wind speed of $55.6 \mathrm{~m} \mathrm{~s}^{-1}$ are used to maximize the correlation between the GVAR counts in a forecast-time cell (in the light gray box in both panels) and the offset cell. This search radius is represented by the darker and larger gray box in the right panel. The correlation-maximizing offset cell is bounded by an offwhite box in the right panel, with the advection vector shown by the arrowhead near the center of the box.

faster than the maximum speed of a cumulus cloud, were searched. The displacement vector between the original block and the pixel-offset block, which maximized the lagged cross correlation, is taken to be the frozen advection vector direction for cloud movement toward the original cell. This process is visualized for an example cell in Fig. 4.

This satellite-based cloud-track method has been used in solar power forecasting (Perez et al. 2010; Law et al. 2014). A similar method, using total sky imagers, is commonly used in very short-range solar power forecasting (e.g., Chow et al. 2011; Huang et al. 2013; Marquez and Coimbra 2013; Quesada-Ruiz et al. 2014). This technique for estimating the advection vector of features from satellite imagery has a long history in meteorology (e.g., Bauer 1976; Rodgers et al. 1979; Schmetz et al. 1993; Menzel 2001; Dunion and Velden 2002). When estimating the advection vectors of particles in a fluid flow from a series of images, the method is called particle image velocimetry (e.g., Westerweel 1997). A closely related cloud-track method for forecasting solar power production, interpreting the satellite images as realizations of a random field and using a Monte Carlo simulation to obtain wind vector estimates, was pioneered by Hammer et al. (1999).

Aside from the forecast error resulting from cloud field evolution, errors may result from misspecification of the advection vectors. Le Marshall et al. (2004) studied errors for cloud motion vectors derived from visible wavelength images with $1.25-\mathrm{km}$ resolutions by cloud height, finding a mean magnitude of vector difference of 3.33-4.99 $\mathrm{m} \mathrm{s}^{-1}$, depending on cloud height, over Australia; an earlier study (Le Marshall et al. 1994) had found errors over Australia from infrared images to be $3.9-4.8 \mathrm{~m} \mathrm{~s}^{-1}$. Schmetz et al. (1993) found a similar range of error $\left(2-7 \mathrm{~m} \mathrm{~s}^{-1}\right)$ for cloud-track wind vectors estimated from the Meteosat infrared channel.

A typical source of error in computing motion vectors by finding maximizing lagged cross correlations is the presence of random correlation peaks (Westerweel 1994). To detect these spurious vectors, a local median filter (following Westerweel 1994) was used to test the spatial coherence of the derived wind vectors. For each vector $\mathbf{v}_{0}$, the componentwise median $\mathbf{v}_{m}$ of its $3 \times 3$ neighborhood $\left\{\mathbf{v}_{1}, \ldots, \mathbf{v}_{8}\right\}$ (excluding $\mathbf{v}_{0}$ ) was calculated, and the median of the residuals $r_{i}=\left|\mathbf{v}_{i}-\mathbf{v}_{m}\right|$ denoted by $r_{m}$, was obtained. This median residual [plus some fluctuation tolerance $\varepsilon$, which is taken as 0.1 times the cell size in accordance with the guidelines in Westerweel and Scarano (2005)] is used to normalize the residual of $\mathbf{v}_{0}$ :

$$
r_{0}^{\prime}=\frac{\left|\mathbf{v}_{0}-\mathbf{v}_{m}\right|}{r_{m}+\varepsilon} .
$$

The quantity $\mathbf{v}_{0}$ is nonspurious if this normalized residual is within a threshold value. Westerweel and Scarano (2005) demonstrated that a threshold value of 2 universally identifies the largest $10 \%$ of residuals, and this is the threshold used in this study. If a vector is found to be spurious, it is linearly interpolated using its neighboring cells (if the cell associated with such a spurious vector is along the edge of the domain, additional cells toward the interior of the domain were used to identify the slope).

Observe that if a cell were entirely clear, the correlation between it and any other cell would be infinite because of its counts having a standard deviation of zero. As a result, the percentage of zero-valued pixels for each cell was compared with a fixed threshold: If this percentage was greater than the threshold, the estimated advection vector was set to 0 (akin to an unmasked cell tracking the fixed terrain). If this threshold is too high, random correlation 
peaks are likely to be generated for cells with sufficiently sparse cloud features, while if it is too low, additional cells will have an initially estimated advection vector of 0 . The threshold value was determined using a test dataset (data from September 2014 over the same study area and hours) by minimizing the total number of vectors that were set to 0 after comparing their cells with the threshold, flagged as spurious (determined using the previously described local median test), or having infinite values. The resulting optimal threshold was $96 \%$.

Once the advection vector is estimated, its direction is treated as the frozen advection direction, while its magnitude scales with respect to the lead time used in a forecast. The forecast for a lead time $\tau$ is generated for each cell $b$ at time $t+\tau$ by forecasting the value of each pixel $p$ in $b$ to be the value in pixel $p-(\tau / 15) \hat{\mathbf{v}}_{t}^{b}$ at forecast-issuance time $t$, where $\hat{\mathbf{v}}_{t}^{b}$ is the cloud-track advection vector estimated for $b$ at time $t$. Sample forecasts are included in Fig. S1 of the online supplementary material.

\section{c. Probabilistic estimates of forecast error}

As mentioned in the introduction (section 1), Eq. (1) will not be strictly valid over all forecast lead times, though it may be an excellent approximation for sufficiently small values of $\Delta t$. A probabilistic method for estimating the expected forecast error at the time of forecast would be desirable for operational planning. However, the cloud-track advection-only method utilized in this study is necessarily deterministic. Thus, we will seek to quantify the uncertainty associated with cloud-track advection-only forecasting of a cumulusdominated cloud field by fitting a statistical model for the root-mean-square error (RMSE) of the deterministic forecast for each cell, using quantile regression (see section 2d) to identify a prediction interval for the RMSE, that is, the range within which the forecast's RMSE can be expected to fall a given percentage of the time. This method for extending deterministic forecasts to a pseudoprobabilistic forecast by estimating quantiles is a simplified version of the procedure from Nielsen et al. (2006), there applied to wind energy power forecasting.

To understand the factors that contribute to changes in error magnitude, we begin by decomposing the mean-square error (MSE). While we will work with the RMSE, the MSE is more suitable for decomposition. Following Murphy [1988, Eq. (9)], the MSE of a forecast can be decomposed into

$$
\operatorname{MSE}=\operatorname{Bias}^{2}(F, O)+\operatorname{Var}(F)+\operatorname{Var}(O)-2 \operatorname{Cov}(F, O),
$$

where $F$ is the forecast quantity and $O$ is the observed quantity.
Equation (4) indicates that the skill of a forecast lies in minimizing the bias and maximizing the covariance of the forecast and the observations, with the variances of the forecast and the observations quantifying the difficulty of obtaining an accurate forecast. Intuitively, the variance component of MSE reflects that obtaining an accurate forecast is more difficult for a broken cloud field as opposed to a relatively uniformly clear or overcast field.

While Eq. (4) allows for a postforecast understanding of the relative contributions to forecast error magnitude, it is of less utility if a predictive estimate of forecast error is desired at the time of forecast as the statistics of $O$ are necessarily unknown. To obtain a statistical prediction of the error magnitude only in terms of the statistics of $F$, we will consider in section $3 \mathrm{~b}$ the skill of five statistical models for the RMSE of the advection-only forecast for each lead time. For those models including the forecast hour, the hour is treated as a categorical predictor (data associated with that hour are associated with an indicator variable, altering the intercept) rather than a numerical predictor as it was found that models using the forecast hour as a categorical predictor outperformed models using the forecast hour as a linear predictor. The five statistical models are

1) $\mathrm{RMSE} \sim \operatorname{Mean}(F)$,

2) $\mathrm{RMSE} \sim \operatorname{Mean}(F)+I$ (forecast hour),

3) $\mathrm{RMSE} \sim \operatorname{Mean}(F)+[\operatorname{Mean}(F)]^{2}$,

4) $\mathrm{RMSE} \sim \operatorname{Mean}(F)+\operatorname{Var}(F)$, and

5) $\mathrm{RMSE} \sim \operatorname{Mean}(F)+\operatorname{Var}(F)+I$ (forecast hour).

Model 1 (regressing the error against the mean) will serve as a benchmark model. The diurnal cycle of fairweather cumuli (and the similarity of the correlation trends with respect to forecast hour in Fig. 5) suggest that incorporating the forecast hour as a predictor would improve the accuracy of the error prediction, particularly for longer lead times. As an advection-only forecast of either a uniformly overcast or uniformly clear sky ought to have similar levels of skill, model 3 is quadratic with respect to the forecast mean.

The analysis of Eq. (4) had also suggested that increased cloud field variance should be positively correlated with increased error. Because of this observation, $\operatorname{Var}(F)$ is introduced as a predictor in model 4. It is not a priori clear whether forecast hour is an independent predictor from either the mean or the variance, as the diurnal cycle effect may be observable in other statistics of the forecast cells that are not explicitly dependent on the forecast hour, such as a change in the "typical" variance of the measurements in each cell as cumulus that form early in the day mature, so this model can be compared with models 2 and 3 to help gain an understanding as to which variable fforecast hour, 

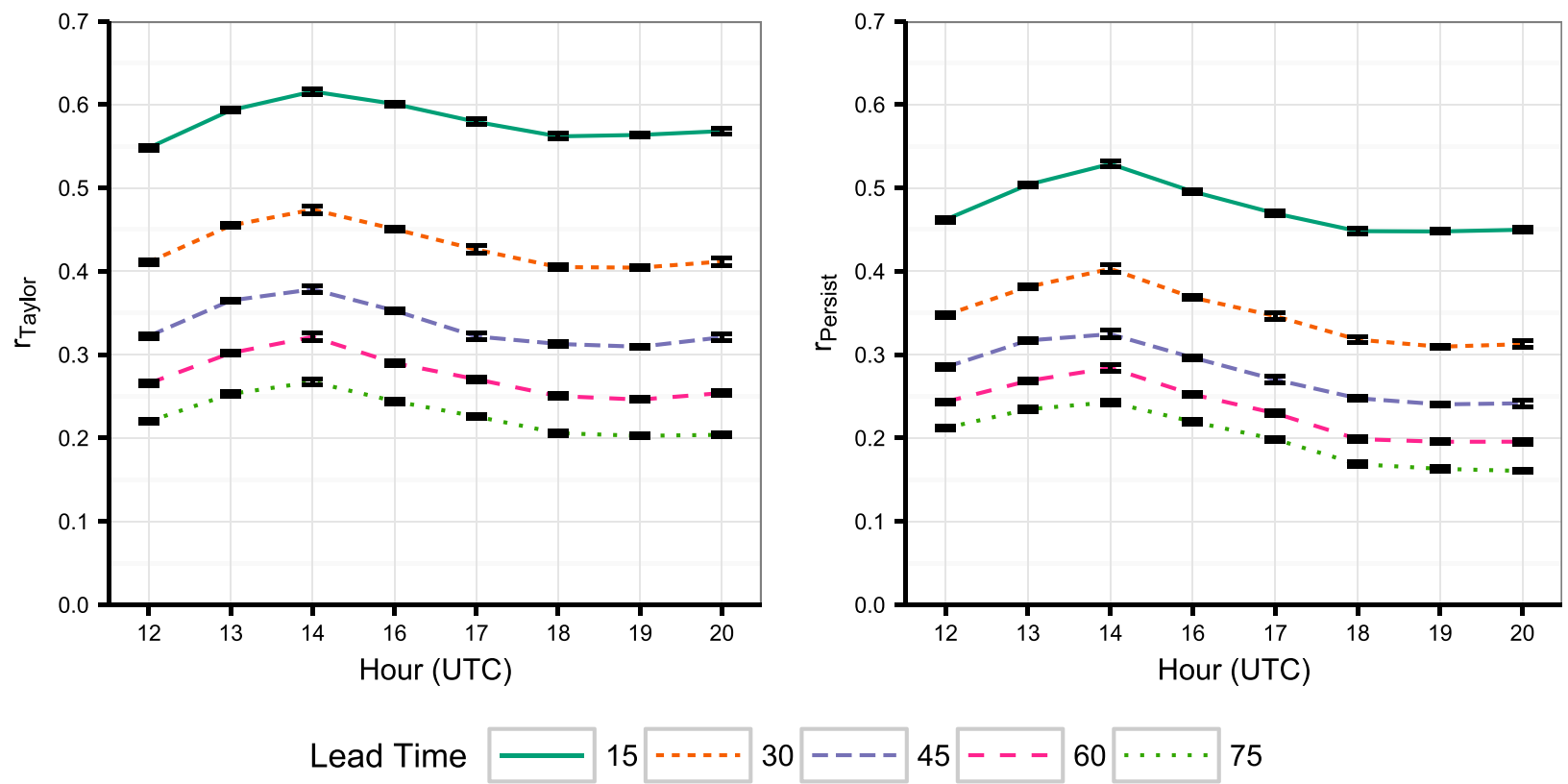

FIG. 5. Mean correlation coefficients between the forecast and observed values for each cell by hour of forecast issuance and forecast lead time ( $\mathrm{min}$ ) for both the (left) Taylor (advection only) forecast and the (right) persistence forecast. Completely clear cells, which composed $7.4 \%$ of the original dataset, were excluded from this calculation because of the zero standard deviation of the contained pixel values. Means and standard errors (as shown by the error bars) were calculated using the Fisher $z$ transformation: $z=\operatorname{atanh}(r)$.

$[\operatorname{Mean}(F)]^{2}$, or $\left.\operatorname{Var}(F)\right\}$ provides the most information relevant to predicting the error of an advection-only forecast. Finally, model 5 incorporates both variance and forecast hour terms to see if these two predictors provide independent information.

\section{d. Quantile regression}

To fully capture the uncertainty associated with a forecast, a full specification of the forecast probability distribution function is ideal. When such a probabilistic method is not possible, one method for turning a deterministic forecast into a probabilistic forecast is to estimate the quantiles of the probability distribution (Taylor and Bunn 1999). These quantiles can be used to forecast a central prediction interval of the modeled quantity, where the central prediction interval with nominal coverage rate $(1-\alpha)$ is the interval centered around the median in which the observation is expected to occur with probability $1-\alpha$. To obtain the central prediction interval of the forecast RMSE corresponding to a nominal coverage rate of $(1-\alpha)$, we infer the quantile regression coefficients for the $\alpha / 2 \%$ and $(1-\alpha) / 2 \%$ quantiles. This technique is well established for wind power forecasts (Bremnes 2006; Nielsen et al. 2006; Møller et al. 2008; Pinson and Kariniotakis 2010; Yan et al. 2015) but has been utilized less for solar power forecasts [though some studies estimate prediction intervals for solar power forecasts under the assumption of normally distributed errors (e.g., Lorenz et al. 2009)]. Some examples of applications of quantile regression to the solar power field are Bacher et al. (2009), Zamo et al. (2014), and Alessandrini et al. (2015).

For a given random variable $X$, the quantile $Q(\nu)$ is defined as the value for which the probability of $X<Q(\nu)$ is $\nu$. In quantile regression, $Q(\nu)$ is estimated using (multiple) linear regression, so that

$$
Q(\nu)=\beta_{0}(\nu)+\beta_{1}(\nu) x_{1}+\cdots+\beta_{k}(\nu) x_{k}
$$

for known predictor variables $x_{i}$ and $i=1, \ldots, k$ and regression coefficients $\beta_{i}(\nu)$ and $i=1, \ldots, k$, which are estimated based on observed values using linear optimization (Koenker and Bassett 1978; Nielsen et al. 2006; Møller et al. 2008). The R package "quantreg" was used to estimate the quantile regression coefficients ( $\mathrm{R}$ Core Team 2015; Koenker 2015). Note that quantile regression, by inferring empirical quantiles, does not assume that the data are normally distributed.

In this work, we will focus on central prediction intervals with nominal coverage rates of $50 \%$ and $90 \%$. In Nielsen et al. (2006), it was observed that estimating the regression coefficients $\beta_{i}(\nu)$ separately for different values of $\nu$ can result in unrealistic results, with lower quantiles crossing higher quantiles. To avoid this scenario, Nielsen et al. (2006) recommend beginning with 

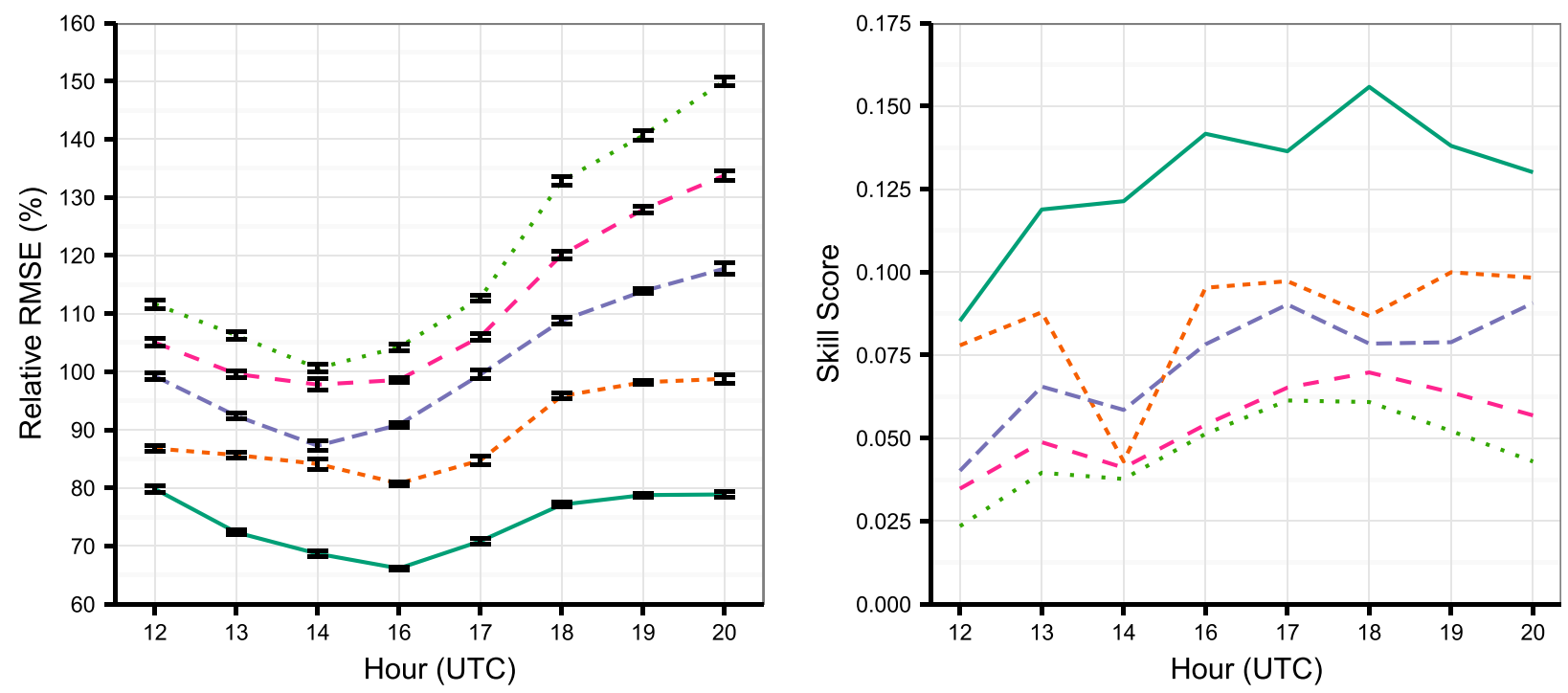

Lead Time $\longrightarrow 75$ = 15

FIG. 6. (left) Relative RMSE (\%) of advection-only forecast across all pixels and (right) the associated skill score relative to a persistence forecast [Eq. (6)] for each lead time by hour of forecast issuance and forecast lead time.

the $50 \%$ quantile and finding upper and lower quantiles under the restriction that they do not cross. In the current study, this potential complication was not encountered.

Following Gneiting and Katzfuss (2014), the two key concepts for validation of a probabilistic forecasting method are calibration and sharpness. Calibration refers to the accuracy of the predictive distributions with respect to the observed values; observations should occur as if they were random draws from a well-calibrated forecast. Sharpness is a measure of only the forecast, reflecting the concentration of the forecast (as a measure of the ability of the forecasting method to distinguish between cases of higher and lower uncertainty). When forecasting prediction intervals, calibration can be assessed by examining whether the empirical coverage rates (the rate at which the data occurred within the interval) on a test set are aligned with the nominal coverage rates, while one measure of sharpness is the mean length of the prediction interval (Bremnes 2006; Nielsen et al. 2006).

To validate the quantile regression predictions, two holdout procedures were used. In the first procedure, for each month, the data corresponding to the first three weeks of each month (day numbers 1-21) were used to train the models, and the data from the remaining days were held out as the test set. The goal of this procedure is to uniformly distribute training and testing data across the season. The second procedure was intended to assess the prediction interval accuracy for a month outside of a training set. The training set consisted of data from June
2014 and July 2014, while the data from August 2014 were held out as the test set, minimizing any potential for autocorrelation effects. The results of these validation procedures for the forecast $50 \%$ and $90 \%$ prediction intervals are given in section $3 \mathrm{c}$.

\section{Results}

\section{a. Advection-only forecasting skill}

The use of images taken at time $t$ and $t-15$ to estimate the wind vector meant that vectors could not be estimated (and hence forecasts issued) during the 1500-1600 UTC hour as only two images were taken during this hour, at 1515 and 1545 UTC. As will be seen from the similarities of the correlation and error curves in Figs. 5 and 6, there is no reason to suppose that this omission will result in a fundamentally different interpretation of the results in this section.

The mean forecast correlation coefficients for each method (Fig. 5) have a similar diurnal cycle regardless of lead time, suggesting that this is a consequence of the meteorological problem (likely due to the reduced number of cumuli and cumulonimbi in the morning relative to the afternoon). For all lead times, the advection-only method yields a higher correlation between forecast and observed cells than does persistence, with a mean (across all cells and all forecast hours) increase in correlation of 0.12 at $15 \mathrm{~min}, 0.08$ at $30 \mathrm{~min}, 0.05$ at $45 \mathrm{~min}, 0.03$ at $60 \mathrm{~min}$, and 0.02 at $75 \mathrm{~min}$. This increase is statistically significant at the $95 \%$ confidence level for 


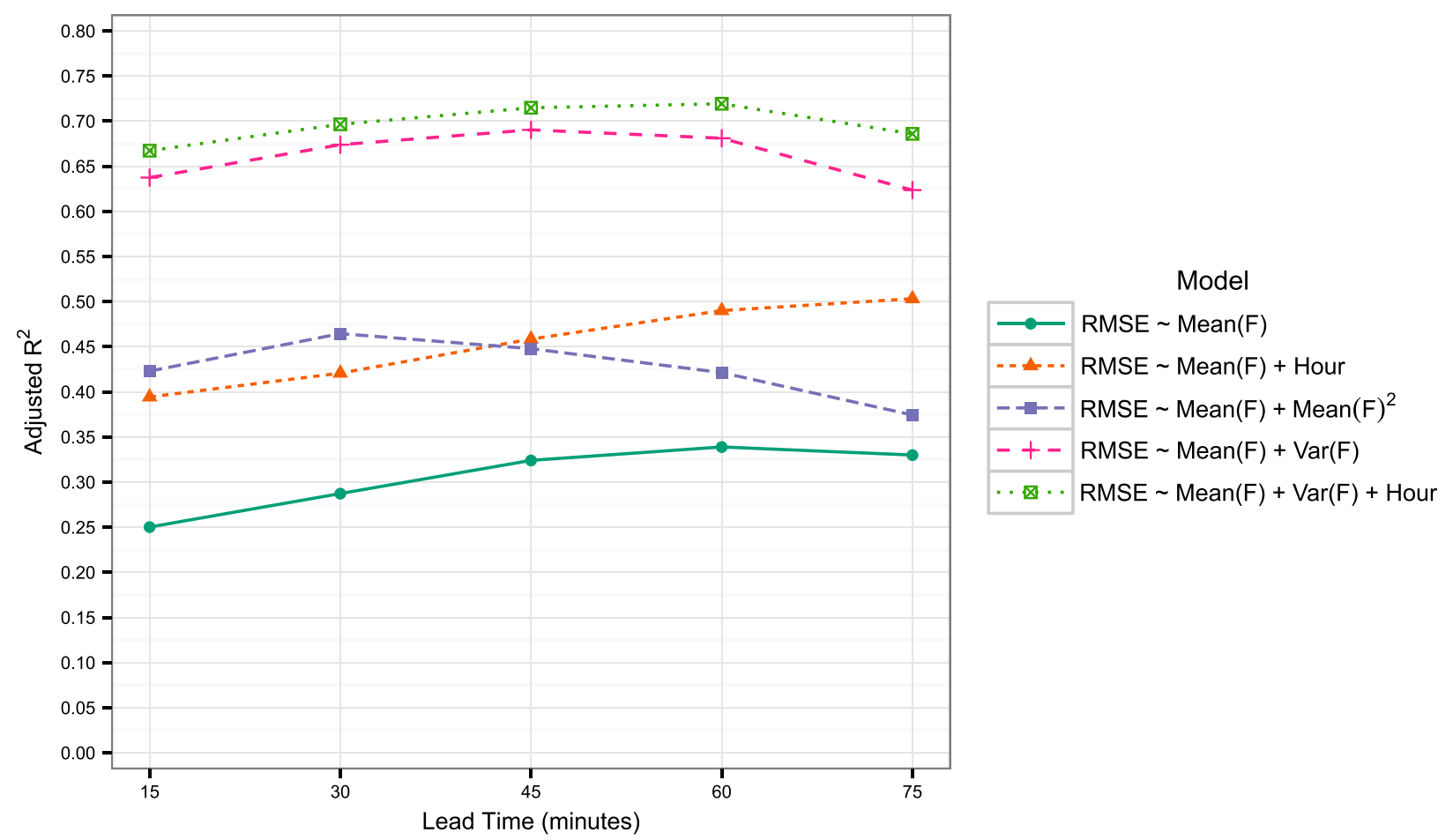

FIG. 7. Plots of adjusted $R^{2}$ with lead time for various statistical models for the forecast RMSE.

lead times through $60 \mathrm{~min}$, showing that a stronger linear relationship exists between forecast and observed cells with the inclusion of advection at these lead times. This improvement is marginal at a lead time of $75 \mathrm{~min}$ as a result of a combination of advancing cloud feature evolution and an increase in the error of the forecast (frozen) advection vector. This observation is consistent with Fig. 1, which suggests the temporal (Lagrangian) scale of cumulus features is $10-60 \mathrm{~min}$.

Figure 6 shows that there is a monotonic increase in the relative RMSE (rRMSE, or RMSE as a percentage of the mean), taken across all pixels, of the advectiononly forecast with lead time for each hour. The rapid increase in rRMSE during the afternoon (particularly from forecast times of 1800-2100 UTC) can be attributed to rapid transition from shallow cumuli to deep precipitating convection, which commonly occurs during these hours during the summer months. While an advection-only method may be able to capture some of this transition with shorter lead times, as the rRMSE is relatively stable during these hours for 15-30-min lead times, the forecasting error resulting from this transition accelerates as the lead times increase. Indeed, only lead times shorter than $30 \mathrm{~min}$ are capable of keeping, on average, the RMSE below $100 \%$ of the observed mean count values for each cell as the 45-min forecast loses this ability in the presence of rapid deep convection.
The skill score in Fig. 6 is calculated relative to the persistence benchmark; following Wilks (2011), the skill is calculated as the relative improvement of the advection-only forecast over persistence:

$$
\text { Skill Score }=\frac{\text { RMSE }_{\text {Persistence }}-\text { RMSE }_{\text {Taylor }}}{\text { RMSE }_{\text {Persistence }}} .
$$

The dip in skill for forecasts issued during the 1400 UTC hour with a 30-min lead time can be attributed to the reduction in sample size resulting from the absence of images in the 1500 UTC hour. Otherwise, the trends are similar to those previously observed, with only the 15 -min lead time forecast resulting in an improvement of at least $10 \%$ over persistence (aside from the 1200 UTC hour). Beyond $30 \mathrm{~min}$, the skill begins to asymptote to a residual skill level, which can be associated with the advection-only forecasting of clear patches or spatially larger, more slowly evolving features. This observation is similar to the results from Cros et al. (2014), which observed a loss of skill prior to $30 \mathrm{~min}$ for a similar forecasting method over the south of France. Here, the skill begins to asymptote beyond $30 \mathrm{~min}$ toward a minimum, as expected from Fig. 1. However, Fig. 6 suggests that this minimum skill is nonzero, indicating that some small amount of residual skill remains in forecasting mesoscale and synoptic-scale features. 
TABLE 1. Regression coefficients, adjusted $R^{2}$, and residual standard errors (SE) for Eq. (7), where the model was fit for forecast lead times of $15,30,45,60$, and $75 \mathrm{~min}$. All coefficients are significant with $p<0.001$.

\begin{tabular}{lcccrr}
\hline \hline Predictor & $15 \mathrm{~min}$ & $30 \mathrm{~min}$ & $45 \mathrm{~min}$ & \multicolumn{1}{c}{$60 \mathrm{~min}$} & $75 \mathrm{~min}$ \\
\hline Mean $(F)$ & $4.43 \times 10^{-2}$ & $4.43 \times 10^{-2}$ & $1.01 \times 10^{-1}$ & $1.35 \times 10^{-1}$ & $1.79 \times 10^{-1}$ \\
$\operatorname{Var}(F)$ & $3.11 \times 10^{-3}$ & $4.02 \times 10^{-3}$ & $4.53 \times 10^{-3}$ & $5.12 \times 10^{-3}$ & $5.84 \times 10^{-3}$ \\
Intercept & 22.85 & 27.29 & 30.16 & 31.62 & 31.15 \\
Adjusted $R^{2}$ & 0.64 & 0.67 & 0.69 & 0.68 & 0.62 \\
Residual SE & 23.42 & 28.01 & 33.23 & 41.40 & 56.6 \\
\hline
\end{tabular}

\section{b. Forecast error model selection}

Figure 6 shows that even at a 15 -min forecast horizon, advection-only forecasting of a cumulus-dominated cloud field in the midlatitudes results in at least a $60 \%$ rRMSE, with this value potentially rising above $100 \%$ for lead times of at least $45 \mathrm{~min}$. This count error should be expected to induce errors in estimates of irradiance, and hence in the generated solar power forecast, though the magnitude of this propagated error will depend on how the cloud field forecast is coupled to the power generation forecast. As such, even if the forecasting method is deterministic, it would be useful to obtain an estimate of the expected forecast error to serve as an approximation of a prediction interval for the forecast and to anticipate the reliability of the forecast. In this section, we focus on modeling the RMSE of a single cell, as once the advection vector field is generated, the cell of interest would be the one forecast to advect over the solar energy conversion site.

The adjusted $R^{2}$ of the five regression models for the RMSE specified in section $2 b$ is graphed in Fig. 7. There is only a slight improvement in the explanatory power of the regression when the hour is explicitly accounted for as a categorical predictor, as can be seen by contrasting the adjusted $R^{2}$ of the RMSE $\sim \operatorname{Mean}(F)$ and RMSE $~$ Mean $(F)+$ Hour models (ranging from 0.12 to 0.27 and 0.18 to 0.29 , respectively), though this effect diminishes slightly with increased lead time.

Following the Murphy (1988) decomposition of the MSE [Eq. (4)], the variance terms in Eq. (4) were interpreted to mean that a broken cloud field was more difficult to forecast than a uniformly clear or overcast field. This intuition is confirmed by Fig. 7, which shows that including the forecast variance along with the forecast mean allows for an improvement in model fit far greater than that from including only the forecast hour with the forecast mean, with the RMSE Mean $(F)+\operatorname{Var}(F)$ attaining adjusted $R^{2}$ values of 0.64 0.67 , depending on lead time. Conversely, including the square of the mean as a predictor improves the model fit relative to that obtained by the mean alone by a much smaller margin. However, there is no statistically significant benefit from including both the forecast variance and the forecast hour as predictors, suggesting that the information contained in the forecast hour is reflected by the forecast variance, so the simplest high-skill model (model 4) is preferred:

$$
\operatorname{RMSE} \sim \operatorname{Mean}(F)+\operatorname{Var}(F) .
$$

The regression coefficients for each lead time are given in Table 1.

\section{c. Quantile regression forecast of error}

To quantify the uncertainty of the predicted forecast error, prediction intervals (with nominal coverage rates of $50 \%$ and $90 \%$ ) for the error were obtained using quantile regression for Eq. (7). As in Eq. (5), we will use the notation $Q(\nu)$ in this section to refer to the prediction at the $\nu$ quantile.

The percentages of RMSEs falling outside or inside of the $50 \%$ and $90 \%$ prediction interval are provided in Tables 2 and 3, respectively. For both nominal coverage rates, the first validation procedure yields a prediction interval that is too wide, while the second procedure

TABLE 2. Calibration rates of validation cells for both quantile regression error $50 \%$ prediction interval validation procedures. Displayed are the percentages of cells with smaller error than forecast by the prediction interval, error within the prediction interval, and error greater than the forecast bounds.

\begin{tabular}{|c|c|c|c|c|c|c|}
\hline \multirow[b]{2}{*}{ Lead time $(\mathrm{min})$} & \multicolumn{3}{|c|}{ Validation 1} & \multicolumn{3}{|c|}{ Validation 2} \\
\hline & $\mathrm{RMSE}<Q_{2} 5$ & $Q_{2} 5 \leq \mathrm{RMSE} \leq Q_{75}$ & $Q_{75} \leq \mathrm{RMSE}$ & $\mathrm{RMSE}<Q_{5}$ & $Q_{5} \leq \mathrm{RMSE} \leq Q_{95}$ & $Q_{95}<\mathrm{RMSE}$ \\
\hline 15 & 21.4 & 58.6 & 20.0 & 24.2 & 51.6 & 24.2 \\
\hline 30 & 22.2 & 58.4 & 19.4 & 25.1 & 51.3 & 23.7 \\
\hline 45 & 22.1 & 58.8 & 19.2 & 23.8 & 51.4 & 24.8 \\
\hline 60 & 21.2 & 59.1 & 19.7 & 23.7 & 51.0 & 25.3 \\
\hline 75 & 20.5 & 59.5 & 20.0 & 22.5 & 51.7 & 25.7 \\
\hline
\end{tabular}


TABLE 3. As in Table 2, but for $90 \%$ prediction interval validation procedures.

\begin{tabular}{|c|c|c|c|c|c|c|}
\hline \multirow[b]{2}{*}{ Lead time (min) } & \multicolumn{3}{|c|}{ Validation 1} & \multicolumn{3}{|c|}{ Validation 2} \\
\hline & $\mathrm{RMSE}<Q_{2} 5$ & $Q 5 \leq \mathrm{RMSE} \leq Q_{95}$ & $Q_{95} \leq \mathrm{RMSE}$ & $\mathrm{RMSE}<Q_{5}$ & $Q_{5} \leq \mathrm{RMSE} \leq Q_{95}$ & $Q_{95}<\mathrm{RMSE}$ \\
\hline 15 & 2.2 & 93.7 & 4.1 & 4.2 & 88.1 & 7.7 \\
\hline 30 & 2.0 & 94.1 & 3.8 & 5.0 & 88.0 & 7.1 \\
\hline 45 & 2.0 & 94.5 & 3.5 & 4.3 & 88.8 & 6.9 \\
\hline 60 & 1.8 & 95.0 & 3.2 & 4.3 & 89.2 & 6.5 \\
\hline 75 & 1.8 & 95.4 & 2.8 & 4.0 & 89.8 & 6.2 \\
\hline
\end{tabular}

yields a reasonably well-calibrated model for the central prediction interval.

The mean prediction interval lengths are shown in Table 4. These lengths monotonically increase with respect to increased lead time, as we would expect given the additional sources of error (increased error in advection vector estimation and additional time for cloud feature evolution). The second validation procedure produces intervals that are slightly sharper than the first, though it is not obvious if these differences are statistically significant.

\section{Conclusions}

We have shown that an advection-only (e.g., assuming Taylor's hypothesis) approach to forecasting cumulusdominated cloud fields in the midlatitudes loses skill at forecasting cumulus-scale features prior to a 60 -min lead time, with the skill beginning to asymptote beyond $30 \mathrm{~min}$. This finding has implications for solar power forecasting methods that rely on advection-only cloud field forecasting: Errors in the forecast cloud field will induce errors in the subsequent radiative transfer forecast. In particular, the method suffers from errors around $100 \%$ of the mean for lead times longer than $30 \mathrm{~min}$. As a result, the use of these frozen advection vectors obtained by correlation-maximization methods for point forecasting the location and radiance of broken cumulus cloud fields cannot be recommended beyond $30 \mathrm{~min}$, let alone the hour recommended by Ela et al. (2013). The more sophisticated probabilistic advection vector-estimation technique used by Hammer et al.
(1999) may be more acceptable over these time scales, though it is not clear what cloud regimes were represented in that study (an important consideration in light of Fig. 1).

This observation is consistent with the data collected by Fujita (1981), visualized in Fig. 1, which indicate that cumulus cloud evolution occurs over 10-60-min time scales. Our results suggest that after 30-45 min, enough evolution has occurred for cumulus-dominated cloud fields that advection-only methods will misforecast mesoscale and submesoscale features. This further indicates that methods to forecast solar power using frozen advection vectors should take into account the cloud regime characterizing the features of interest for a particular forecast, both operationally and when quantifying error, as the forecast skill will likely not decay uniformly with increasing lead time across all cloud regimes. We would expect analogous bounds on lead time to hold for forecasting the larger-scale features depicted in Fig. 1, and work is underway to investigate the specific nature of these limits for features of varying spatiotemporal scales. If a similar pattern is found, it would suggest that solar power forecasting could be optimized with respect to forecast frequency and lead time horizon based on the prevailing meteorological regime: highly convective regimes require more frequent and shorterterm forecasts, while synoptic-scale regimes are more persistent. A similar study could also be undertaken for smaller time and space scales using total sky imager data. It will also be of interest to observe the impact of improved spatial resolution of future imagers on forecasting methods that utilize advection vectors that

TABLE 4. Mean prediction interval lengths for the $50 \%$ and $90 \%$ prediction interval validation procedures. The lengths should be interpreted as ranges of 10 -bit GVAR counts $(0-1024)$

\begin{tabular}{|c|c|c|c|c|}
\hline \multirow[b]{2}{*}{ Lead time (min) } & \multicolumn{2}{|c|}{ Validation 1} & \multicolumn{2}{|c|}{ Validation 2} \\
\hline & $50 \%$ interval & $90 \%$ interval & $50 \%$ interval & $90 \%$ interval \\
\hline 15 & 32.9 & 76.4 & 32.3 & 76.3 \\
\hline 30 & 38.7 & 93.0 & 38.0 & 90.9 \\
\hline 45 & 41.2 & 107.2 & 40.2 & 105.1 \\
\hline 60 & 43.4 & 122.5 & 41.9 & 118.2 \\
\hline 75 & 45.4 & 136.5 & 44.0 & 132.4 \\
\hline
\end{tabular}


TABLE A1. Table of nomenclature.

\begin{tabular}{ll}
\hline \hline Variable & \multicolumn{1}{c}{ Description } \\
\hline$q$ & Forecast quantity function \\
$t$ & Time \\
$\mathbf{p}$ & Spatial position vector \\
$\tau$ & Forecast lead time \\
$\mathbf{v}, \hat{\mathbf{v}}$ & Advection vector \\
$b$ & Forecast cell \\
$p$ & Pixel \\
MSE & Mean-square error \\
$F$ & Forecast quantity \\
$O$ & Observed quantity \\
$1-\alpha$ & Nominal coverage of prediction interval \\
$X$ & Random variable forecast using regression \\
$Q(\nu)$ & $\nu$ quantile of $X$ \\
$x_{i}$ & Prediction variables for $X$ \\
$\beta_{i}$ & Regression coefficients for $X$ \\
\hline
\end{tabular}

maximize lagged cross correlations, such as the visible channel imager on the GOES-R satellite, which has a spatial resolution of $0.5 \mathrm{~km}$ (Schmit et al. 2005), as this should allow for more effective tracking of smaller features.

While the skill of the advection-only method approaches that of persistence as lead times approach $60 \mathrm{~min}$, the use of advection-only methods may be appealing for certain operational uses because of their speed. With the knowledge that the error associated with these methods will be significant, an understanding of the potential error is important for planning, such as committing additional power generators to compensate for a shortfall in expected solar power generation. A statistical model for the RMSE was built by examining the Murphy decomposition for that error, finding that the variance of the forecast cell is an important predictor of the advection-only forecast error. A method for converting this deterministic estimate to a probabilistic estimate via quantile regression was used to construct $50 \%$ and $90 \%$ prediction intervals, though any similar interval could be constructed by using the appropriate quantiles. It is likely that the validation procedure involving the use of previously observed months on a later month (which was well calibrated) is more reflective of potential operational use. While a more intrinsically probabilistic method would be ideal, quantile regression should be sufficiently robust for extending point forecasts of cloud counts or derived quantities, such as radiance, albedo, or brightness.

Acknowledgments. The authors thank Dr. Laura Hinkelman for her helpful comments and ideas regarding the use of satellite imagery, Dr. Tyler McCandless for his advice on working with Taylor's hypothesis, and
Dr. Joseph Ranalli for pointing out the connection between the cloud-track wind estimation method and particle image velocimetry.

\section{APPENDIX}

\section{Mathematical Notation and Abbreviations Used in this Work}

A table of nomenclature is provided in Table A1.

\section{REFERENCES}

Alessandrini, S., L. Delle Monache, S. Sperati, and G. Cervone, 2015: An analog ensemble for short-term probabilistic solar power forecast. Appl. Energy, 157, 95-110, doi:10.1016/ j.apenergy.2015.08.011.

Bacher, P., H. Madsen, and H. A. Nielsen, 2009: Online short-term solar power forecasting. Sol. Energy, 83, 1772-1783, doi:10.1016/ j.solener.2009.05.016.

Bauer, K. G., 1976: A comparison of cloud motion winds with coinciding radiosonde winds. Mon. Wea. Rev., 104, 922-931, doi:10.1175/1520-0493(1976)104<0922:ACOCMW>2.0.CO;2.

Bremnes, J. B., 2006: A comparison of a few statistical models for making quantile wind power forecasts. Wind Energy, 9, 3-11, doi:10.1002/we.182.

Burghelea, T., E. Segre, and V. Steinberg, 2005: Validity of the Taylor hypothesis in a random spatially smooth flow. Phys. Fluids, 17, 103101, doi:10.1063/1.2077367.

Cano, D., J. M. Monget, M. Albuisson, H. Guillard, N. Regas, and L. Wald, 1986: A method for the determination of the global solar radiation from meteorological satellite data. Sol. Energy, 37, 31-39, doi:10.1016/0038-092X(86)90104-0.

Chow, C. W., B. Urquhart, M. Lave, A. Dominguez, J. Kleissl, J. Shields, and B. Washom, 2011: Intra-hour forecasting with a total sky imager at the UC San Diego solar energy testbed. Sol. Energy, 85, 2881-2893, doi:10.1016/j.solener.2011.08.025.

_ S. Belongie, and J. Kleissl, 2015: Cloud motion and stability estimation for intra-hour solar forecasting. Sol. Energy, 115, 645-655, doi:10.1016/j.solener.2015.03.030.

Cox, D. R., and V. Isham, 1988: A simple spatial-temporal model of rainfall. Proc. Roy. Soc., 415A, 317-328, doi:10.1098/ rspa.1988.0016.

Cros, S., O. Liandrat, N. Sébastien, and N. Schmutz, 2014: Extracting cloud motion vectors from satellite images for solar power forecasting. 2014 IEEE Geoscience and Remote Sensing Symp., Quebec City, QC, Canada, Institute of Electrical and Electronics Engineers, 4123-4126, doi:10.1109/IGARSS.2014.6947394.

Del Álamo, J. C., and J. Jiménez, 2009: Estimation of turbulent convection velocities and corrections to Taylor's approximation. J. Fluid Mech., 640, 5-26, doi:10.1017/S0022112009991029.

Dennis, D. J. C., and T. B. Nickels, 2008: On the limitations of Taylor's hypothesis in constructing long structures in a turbulent boundary layer. J. Fluid Mech., 614, 197-206, doi:10.1017/S0022112008003352.

Dunion, J. P., and C. S. Velden, 2002: Application of surfaceadjusted GOES low-level cloud-drift winds in the environment of Atlantic tropical cyclones. Part I: Methodology and validation. Mon. Wea. Rev., 130, 1333-1346, doi:10.1175/ 1520-0493(2002)130<1333:AOSAGL>2.0.CO;2. 
Eber, K., and D. Corbus, 2013: Hawaii solar integration study: Executive summary. National Renewable Energy Laboratory Tech. Rep. NREL/TP-5500-57215, 28 pp. [Available online at http://www.nrel.gov/docs/fy13osti/57215.pdf.]

Ela, E., V. Diakov, E. Ibanez, and M. Heaney, 2013: Impacts of variability and uncertainty in solar photovoltaic generation at multiple timescales. National Renewable Energy Laboratory Tech. Rep. NREL/TP-5500-58274, 34 pp. [Available online at http://www.nrel.gov/docs/fy13osti/58274.pdf.]

Früwirth-Schnatter, S., 2006: Finite Mixture and Markov Switching Models. Springer-Verlag, $494 \mathrm{pp}$.

Fujita, T. T., 1981: Tornadoes and downbursts in the context of generalized planetary scales. J. Atmos. Sci., 38, 1511-1534, doi:10.1175/1520-0469(1981)038<1511:TADITC >2.0.CO;2.

Gifford, F., 1956: The relation between space and time correlations in the atmosphere. J. Meteor., 13, 289-294, doi:10.1175/ 1520-0469(1956)013<0289:TRBSAT >2.0.CO;2.

Gneiting, T., and M. Katzfuss, 2014: Probabilistic forecasting. Annu. Rev. Stat. Appl., 1, 125-151, doi:10.1146/ annurev-statistics-062713-085831.

Gupta, V. K., and E. Waymire, 1987: On Taylor's hypothesis and dissipation in rainfall. J. Geophys. Res., 92, 9657-9660, doi:10.1029/JD092iD08p09657.

Hammer, A., D. Heinemann, E. Lorenz, and B. Lückehe, 1999: Short-term forecasting of solar radiation: A statistical approach using satellite data. Sol. Energy, 67, 139-150, doi:10.1016/S0038-092X(00)00038-4.

Higgins, C. W., M. Froidevaux, V. Simeonov, N. Vercauteren, C. Barry, and M. B. Parlange, 2012: The effect of scale on the applicability of Taylor's frozen turbulence hypothesis in the atmospheric boundary layer. Bound.-Layer Meteor., 143, 379_ 391, doi:10.1007/s10546-012-9701-1.

Huang, H., J. Xu, Z. Peng, S. Yoo, D. Yu, D. Huang, and H. Qin, 2013: Cloud motion estimation for short term solar irradiation prediction. 2013 IEEE Int. Conf. on Smart Grid Communications, Vancouver, BC, Canada, Institute of Electrical and Electronics Engineers, 696-701, doi:10.1109/SmartGridComm.2013.6688040.

Koenker, R., 2015: quantreg: Quantile Regression, version 5.19. R package. [Available online at https://CRAN.R-project.org/ package $=$ quantreg.]

_ 46, 33-50, doi:10.2307/1913643.

Law, E. W., A. A. Prasad, M. Kay, and R. A. Taylor, 2014: Direct normal irradiance forecasting and its application to concentrated solar thermal output forecasting-A review. Sol. Energy, 108, 287-307, doi:10.1016/j.solener.2014.07.008.

Le Marshall, J., N. Pescod, B. Seaman, G. Mills, and P. Stewart, 1994: An operational system for generating cloud-drift winds in the Australian region and their impact on numerical weather prediction. Wea. Forecasting, 9, 361-370, doi:10.1175/ 1520-0434(1994)009<0361:AOSFGC > 2.0.CO;2.

—, A. Rea, L. Leslie, R. Seecamp, and M. Dunn, 2004: Error characterisation of atmospheric motion vectors. Aust. Meteor. Mag., 53, 123-131. [Available online at http://citeseerx.ist.psu.edu/ viewdoc/download?doi $=10.1 .1 .222 .8275 \&$ rep $=$ rep1\&type $=$ pdf.]

Li, B., A. Murthi, K. P. Bowman, G. R. North, M. G. Genton, and M. Sherman, 2009: Statistical tests of Taylor's hypothesis: An application to precipitation fields. J. Hydrometeor., 10, 254 265, doi:10.1175/2008JHM1009.1.

Lorenz, E., J. Hurka, D. Heinemann, and H. G. Beyer, 2009: Irradiance forecasting for the power prediction of grid-connected photovoltaic systems. IEEE J. Sel. Top. Appl. Earth Obs. Remote Sens., 2, 2-10, doi:10.1109/JSTARS.2009.2020300.
Marquez, R., and C. F. M. Coimbra, 2013: Intra-hour DNI forecasting based on cloud tracking image analysis. Sol. Energy, 91, 327-336, doi:10.1016/j.solener.2012.09.018.

Mathiesen, P., C. Collier, and J. Kleissl, 2013: A high-resolution, cloud-assimilating numerical weather prediction model for solar irradiance forecasting. Sol. Energy, 92, 47-61, doi:10.1016/ j.solener.2013.02.018.

McLachlan, G., and D. Peel, 2000: Finite Mixture Models. WileyInterscience, $419 \mathrm{pp}$.

Menzel, W. P., 2001: Cloud tracking with satellite imagery: From the pioneering work of Ted Fujita to the present. Bull. Amer. Meteor. Soc., 82, 33-47, doi:10.1175/1520-0477(2001)082<0033: CTWSIF $>2.3 . \mathrm{CO} ; 2$

Miller, S., M. Rogers, A. Heidinger, I. Laszlo, and M. Sengupta, 2012: Cloud advection schemes for short-term satellitebased insolation forecasts. World Renewable Energy Forum 2012, Denver, CO, American Solar Energy Society, 1963 1967.

Mizuno, T., and H. A. Panofsky, 1975: The validity of Taylor's hypothesis in the atmospheric surface layer. Bound.-Layer Meteor., 9, 375-380, doi:10.1007/BF00223388.

Møller, J. K., H. A. Nielsen, and H. Madsen, 2008: Time-adaptive quantile regression. Comput. Stat. Data Anal., 52, 1292-1303, doi:10.1016/j.csda.2007.06.027.

Murphy, A. H., 1988: Skill scores based on the mean square error and their relationships to the correlation coefficient. Mon. Wea. Rev., 116, 2417-2424, doi:10.1175/1520-0493(1988)116<2417: $\mathrm{SSBOTM}>2.0 . \mathrm{CO} ; 2$.

Nielsen, H. A., H. Madsen, and T. S. Nielsen, 2006: Using quantile regression to extend an existing wind power forecasting system with probabilistic forecasts. Wind Energy, 9, 95-108, doi:10.1002/we. 180 .

Perez, R., P. Ineichen, K. Moore, M. Kmiecik, C. Chain, R. George, and F. Vignola, 2002: A new operational model for satellitederived irradiances: Description and validation. Sol. Energy, 73, 307-317, doi:10.1016/S0038-092X(02)00122-6.

—, S. Kivalov, J. Schlemmer, K. Hemker Jr., D. Renné, and T. E. Hoff, 2010: Validation of short and medium term operational solar radiation forecasts in the US. Sol. Energy, 84, 2161-2172, doi:10.1016/j.solener.2010.08.014.

Pinson, P., and G. Kariniotakis, 2010: Conditional prediction intervals of wind power generation. IEEE Trans. Power Syst., 25, 1845-1856, doi:10.1109/TPWRS.2010.2045774.

Pollard, B. D., S. Khanna, S. J. Frasier, J. C. Wyngaard, D. W. Thomson, and R. E. McIntosh, 2000: Local structure of the convective boundary layer from a volume-imaging radar. J. Atmos. Sci., 57, 2281-2296, doi:10.1175/1520-0469(2000)057<2281: LSOTCB $>2.0 . \mathrm{CO} ; 2$.

Powell, D. C., and C. E. Elderkin, 1974: An investigation of the application of Taylor's hypothesis to the atmospheric boundary layer turbulence. J. Atmos. Sci., 31, 990-1002, doi:10.1175/ 1520-0469(1974)031<0990:AIOTAO > 2.0.CO;2.

Quesada-Ruiz, S., Y. Chu, J. Tovar-Pescador, H. T. C. Pedro, and C. F. M. Coimbra, 2014: Cloud-tracking methodology for intra-hour DNI forecasting. Sol. Energy, 102, 267-275, doi:10.1016/j.solener.2014.01.030.

Rayl, J., G. S. Young, and J. R. S. Brownson, 2013: Irradiance cospectrum analysis: Tools for decision support and technological planning. Sol. Energy, 95, 364-375, doi:10.1016/ j.solener.2013.02.029.

R Core Team, 2015: R: A language and environment for statistical computing. R Foundation for Statistical Computing. [Available online at https://www.R-project.org/.] 
Rodgers, E., R. C. Gentry, W. Shenk, and V. Oliver, 1979: The benefits of using short-interval satellite images to derive winds for tropical cyclones. Mon. Wea. Rev., 107, 575-584, doi:10.1175/1520-0493(1979)107<0575:TBOUSI > 2.0.CO;2.

Schmetz, J., K. Holmlund, J. Hoffman, B. Strauss, B. Mason, V. Gaertner, A. Koch, and L. Van De Berg, 1993: Operational cloud-motion winds from Meteosat infrared images. J. Appl. Meteor., 32, 1206-1225, doi:10.1175/1520-0450(1993)032<1206: OCMWFM $>2.0 . \mathrm{CO} ; 2$.

Schmit, T. J., M. M. Gunshor, W. P. Menzel, J. J. Gurka, J. Li, and A. S. Bachmeier, 2005: Introducing the next-generation Advanced Baseline Imager on GOES-R. Bull. Amer. Meteor. Soc., 86, 1079-1096, doi:10.1175/BAMS-86-8-1079.

Taylor, G. I., 1938: The spectrum of turbulence. Proc. Roy. Soc., 164A, 476-490, doi:10.1098/rspa.1938.0032.

Taylor, J. W., and D. W. Bunn, 1999: A quantile regression approach to generating prediction intervals. Manage. Sci., 45, 225-237, doi:10.1287/mnsc.45.2.225.

Von Meier, A., 2011: Integration of renewable generation in California: Coordination challenges in time and space. 11th Int. Conf. on Electrical Power Quality and Utilisation, Lisbon, Portugal, Institute of Electrical and Electronics Engineers, 768-773, doi:10.1109/EPQU.2011.6128888.

Wacongne, S., and A. Babiano, 1982: Space-time turbulent characteristics in the atmospheric surface layer. Bound.-Layer Meteor., 24, 429-450, doi:10.1007/BF00120732.

Westerweel, J., 1994: Efficient detection of spurious vectors in particle image velocimetry data. Exp. Fluids, 16, 236-247, doi:10.1007/BF00206543.
_ 1997: Fundamentals of digital particle image velocimetry. Meas. Sci. Technol., 8, 1379-1392, doi:10.1088/0957-0233/8/ $12 / 002$.

- , and F. Scarano, 2005: Universal outlier detection for PIV data. Exp. Fluids, 39, 1096-1100, doi:10.1007/ s00348-005-0016-6.

Wilczek, M., H. Xu, and Y. Narita, 2014: A note on Taylor's hypothesis under large-scale flow variation. Nonlinear Processes Geophys., 21, 645-649, doi:10.5194/ npg-21-645-2014.

Wilks, D. S., 2011: Statistical Methods in the Atmospheric Sciences. 3rd ed. Elsevier, $676 \mathrm{pp}$.

Yan, J., Y. Liu, S. Han, Y. Wang, and S. Feng, 2015: Reviews on uncertainty analysis of wind power forecasting. Renewable Sustainable Energy Rev., 52, 1322-1330, doi:10.1016/ j.rser.2015.07.197.

Yang, H., B. Kurtz, D. Nguyen, B. Urquhart, C. W. Chow, M. Ghonima, and J. Kleissl, 2014: Solar irradiance forecasting using a ground-based sky imager developed at UC San Diego. Sol. Energy, 103, 502-524, doi:10.1016/ j.solener.2014.02.044.

Zamo, M., O. Mestre, P. Arbogast, and O. Pannekoucke, 2014: A benchmark of statistical regression methods for short-term forecasting of photovoltaic electricity production. Part II: Probabilistic forecast of daily production. Sol. Energy, 105, 804-816, doi:10.1016/j.solener.2014.03.026.

Zawadzki, I. I., 1973: Statistical properties of precipitation patterns. J. Appl. Meteor., 12, 459-472, doi:10.1175/ 1520-0450(1973)012<0459:SPOPP $>2.0 . \mathrm{CO} ; 2$. 